\title{
A variant of Yano's extrapolation theorem on Hardy spaces
}

\author{
ODYSSEAS BAKAS
}

\begin{abstract}
In this note, a variant of Yano's classical extrapolation theorem for sublinear operators acting on analytic Hardy spaces over the torus is obtained.
\end{abstract}

Mathematics Subject Classification. Primary 30H10, 42B35, 46B70; Secondary 42B25.

Keywords. Extrapolation, Orlicz spaces, Hardy spaces on the torus, Littlewood-Paley square function.

1. Introduction. If $(X, \mu)$ and $(Y, \nu)$ are two finite measure spaces and $T$ is a sublinear operator such that there exist constants $C_{0}, r>0$ satisfying

$$
\sup _{\|g\|_{L^{p}(X)}=1}\|T(g)\|_{L^{p}(Y)} \leq C_{0}(p-1)^{-r}
$$

for every $1<p \leq 2$, then a classical theorem of S. Yano [27] asserts that

$$
\|T(f)\|_{L^{1}(Y)} \leq A+B \int_{X}|f(x)| \log ^{r}(1+|f(x)|) d \mu(x)
$$

for all simple functions $f$ on $X$, where $A, B>0$ are constants depending only on $C_{0}, r, \mu(X)$, and $\nu(Y)$. See also [30, Chapter XII, Theorem 4.41].

This note focuses on a version of the aforementioned extrapolation theorem of Yano for sublinear operators acting on functions belonging to analytic Hardy spaces over the torus. Namely, it is shown here that if $T$ is a sublinear operator acting on functions defined over the torus such that its operator norm from $\left(H^{p}(\mathbb{T}),\|\cdot\|_{L^{p}(\mathbb{T})}\right)$ to $\left(L^{p}(\mathbb{T}),\|\cdot\|_{L^{p}(\mathbb{T})}\right)$ behaves like $(p-1)^{-r}$ as $p \rightarrow 1^{+}$, then $T$ satisfies an inequality analogous to (1.2) for functions in analytic Hardy spaces, see Theorem 1 below.

The study of such variants of Yano's extrapolation theorem in the present note is motivated by some classical results of S. Pichorides [23] and A. Zygmund [29, Theorem 8] on mapping properties of the Littlewood-Paley square function 
$S$ "near" $H^{1}(\mathbb{T})$. Recall that given a trigonometric polynomial $f$ on $\mathbb{T}$, the classical Littlewood-Paley square function $S(f)$ of $f$ is defined as

$$
S(f):=\left(\sum_{k \in \mathbb{Z}}\left|\Delta_{k}(f)\right|^{2}\right)^{1 / 2},
$$

where $\Delta_{0}(f)(\theta):=\widehat{f}(0), \theta \in \mathbb{T}$ and for $k \in \mathbb{N}$,

$$
\Delta_{k}(f)(\theta):=\sum_{n=2^{k-1}}^{2^{k}-1} \widehat{f}(n) e^{i 2 \pi n \theta} \text { and } \Delta_{-k}(f)(\theta):=\sum_{n=-2^{k}+1}^{-2^{k-1}} \widehat{f}(n) e^{i 2 \pi n \theta}
$$

for $\theta \in \mathbb{T}$. In [29], Zygmund showed that for every function $f \in H^{1}(\mathbb{T})$, one has

$$
\|S(f)\|_{L^{1}(\mathbb{T})} \lesssim 1+\int_{\mathbb{T}}|f(\theta)| \log (1+|f(\theta)|) d \theta \quad\left(f \in H^{1}(\mathbb{T})\right)
$$

and in [23], Pichorides showed that

$$
\sup _{\substack{g \in H^{p}(\mathbb{T}): \\\|g\|_{L^{p}}(\mathbb{T})}}\|S(g)\|_{L^{p}(\mathbb{T})} \sim(p-1)^{-1}
$$

as $p \rightarrow 1^{+}$. Both of these results were originally proved by using one-dimensional complex-analytic techniques such as the canonical factorisation of Hardy spaces on the torus. Recently, in [2], the aforementioned results of Pichorides and Zygmund were extended to higher dimensions in a unified way using "real-variable" techniques. In particular, it is shown in [2] that both (1.3) and (1.4) can be obtained by using a result of T. Tao and J. Wright on endpoint mapping properties of Marcinkiewicz multiplier operators [26] together with a "Marcinkiewicz-type" interpolation argument for $H^{p}(\mathbb{T})$ spaces due to S. Kislyakov and Q. Xu [17], see also [6]. Therefore, motivated by the above remarks, one is naturally led to ask whether it is possible to deduce the result of Zygmund (1.3) directly from that of Pichorides (1.4) by using some "real-variable" approach or, even more generally, whether a general extrapolation theorem for sublinear operators acting on $H^{p}(\mathbb{T})$ spaces holds true. The following theorem shows that this is indeed the case.

Theorem 1. Let $T$ be a sublinear operator acting on functions defined over $\mathbb{T}$, namely for all measurable functions $f, g$ on $\mathbb{T}$ and each complex number $\alpha$, one has $|T(f+g)| \leq|T(f)|+|T(g)|$ and $|T(\alpha f)|=|\alpha||T(f)|$.

If there exist constants $C_{0}, r>0$ such that

$$
\sup _{\substack{g \in H^{p}(\mathbb{T}): \\\|g\|_{L^{p}(\mathbb{T})}=1}}\|T(g)\|_{L^{p}(\mathbb{T})} \leq C_{0}(p-1)^{-r}
$$

for every $1<p \leq 2$, then there exists a constant $D>0$, depending only on $C_{0}, r$, such that

$$
\|T(f)\|_{L^{1}(\mathbb{T})} \leq D\|f\|_{L \log ^{r} L(\mathbb{T})}
$$

for every analytic trigonometric polynomial $f$ on $\mathbb{T}$. 
We remark that Theorem 1 can be deduced from already existing results, even though that, to the best of our knowledge, such an explicit statement of an extrapolation-type result for analytic Hardy spaces has not appeared before. More specifically, Theorem 1 follows from a general result of M. Carro and J. Martín on extrapolation of operators acting on compatible couples of Banach spaces [11] (see also the work of B. Jawerth and M. Milman [13]) combined with the $K$-closedness of $\left(H^{1}(\mathbb{T}), H^{\infty}(\mathbb{T})\right)$ with respect to $\left(L^{1}(\mathbb{T}), L^{\infty}(\mathbb{T})\right)$, a result due to Peter Jones [14]. See also Section 3.2.

However, one can give a more elementary and direct proof of Theorem 1, avoiding deep techniques of abstract interpolation, such as the $K$-divisibility theorem [10, Theorem 3.2.7], used in [11]. Indeed, our purpose here is to present a self-contained proof of Theorem 1 that can be obtained by employing ideas from Yano's original approach [27] combined with the so-called "analytic decomposition of unity", a technique due to Kislyakov [15], which only uses the $L^{2}(\mathbb{T})$-boundedness of the Hilbert transform. At this point, it is worth mentioning that the main idea in Yano's paper is to decompose a given function $f$ as

$$
f=\sum_{n \in \mathbb{N}_{0}} f_{n},
$$

where $f_{0}=\chi_{\{|f|<1\}} f$ and $f_{n}=\chi_{\left\{2^{n-1} \leq|f|<2^{n}\right\}} f$ (for $n \in \mathbb{N}$ ) and then apply the assumption (1.1) to each $f_{n}$ separately for $p=p_{n}=1+1 /(n+1)$, noting that $f_{n} \in L^{p_{n}}(\mathbb{T})$ for $n \in \mathbb{N}_{0}$. Unfortunately, given a function $f \in H^{1}(\mathbb{T})$, if one defines $f_{n}$ as above, then $f_{n}$ are not necessarily in the analytic Hardy space $H^{p_{n}}(\mathbb{T})$ anymore and hence, one cannot apply (1.5) to $f_{n}$. To surpass this difficulty, the idea is to use Kislyakov's "analytic decomposition of unity" of $f=\sum_{n} \widetilde{f}_{n}[15]$, see also J. Bourgain's paper [7]. In particular, each $\widetilde{f}_{n}$ may be regarded as an appropriate " $H^{\infty}$-replacement" of $f_{n}$ in the sense that $\widetilde{f}_{n} \in H^{\infty}(\mathbb{T}), f=\sum_{n} \widetilde{f}_{n}$, and each $\widetilde{f}_{n}$ "essentially behaves like" $f_{n}, f_{n}$ being as above. The details of the direct approach described above are given in Section 2. In Section 3, we make some additional remarks related to the present work.

Notation. We denote the set of integers by $\mathbb{Z}$. The set of natural numbers is denoted by $\mathbb{N}$ and the set of non-negative integers is denoted by $\mathbb{N}_{0}$.

We identify functions over the torus $\mathbb{T}$ with functions defined on the set $[0,1)$.

If $f \in L^{1}(\mathbb{T})$ is such that $\operatorname{supp}(\widehat{f})$ is finite, then $f$ is said to be a trigonometric polynomial on $\mathbb{T}$. If $f$ is a trigonometric polynomial on $\mathbb{T}$ such that $\operatorname{supp}(\widehat{f}) \subset \mathbb{N}_{0}$, then we say that $f$ is an analytic trigonometric polynomial on $\mathbb{T}$.

For $1 \leq p \leq \infty$, the analytic Hardy space $H^{p}(\mathbb{T})$ is the space of all functions $f \in L^{p}(\mathbb{T})$ such that $\widehat{f}(n)=0$ when $n<0$. If we equip $H^{p}(\mathbb{T})$ with the $\|\cdot\|_{L^{p}(\mathbb{T})^{-}}$ norm, then $\left(H^{p}(\mathbb{T}),\|\cdot\|_{L^{p}(\mathbb{T})}\right)$ becomes a Banach space. Similarly, for $n \in \mathbb{N}$, one defines $H^{p}\left(\mathbb{T}^{n}\right):=\left\{f \in L^{p}\left(\mathbb{T}^{n}\right): \operatorname{supp}(\widehat{f}) \subset \mathbb{N}_{0}^{n}\right\}, 1 \leq p \leq \infty$. For more details on Hardy spaces we refer the reader to the book [12]. 
For $r>0, L \log ^{r} L(\mathbb{T})$ denotes the class of all measurable functions $f$ on $\mathbb{T}$ satisfying $\int_{\mathbb{T}}|f(\theta)| \log ^{r}(1+|f(\theta)|) d \theta<\infty$. For $f \in L \log ^{r} L(\mathbb{T})$, if we set

$$
\|f\|_{L \log ^{r} L(\mathbb{T})}:=\inf \left\{\lambda>0: \int_{\mathbb{T}} \Phi_{r}\left(\lambda^{-1}|f(\theta)|\right) d \theta \leq 1\right\},
$$

where $\Phi_{r}(x)=x\left([1+\log (x+1)]^{r}-1\right)(x \geq 0)$, then $\|\cdot\|_{L \log ^{r} L(\mathbb{T})}$ is a norm on $L \log ^{r} L(\mathbb{T})$ and, moreover, $\left(L \log ^{r} L(\mathbb{T}),\|\cdot\|_{L \log ^{r} L(\mathbb{T})}\right)$ is a Banach space. For more details on Orlicz spaces, see [18]. It is well-known that

$$
\|f\|_{L \log ^{r} L(\mathbb{T})} \sim \int_{0}^{1} f^{*}(t) \log ^{r}(e / t) d t
$$

where $f^{*}(t):=\inf \{\lambda>0:|\{x \in \mathbb{T}:|f(x)|>\lambda\}| \leq t\}$ is the non-increasing rearrangement of $f$; see e.g. the proof of [3, Lemma 10.1].

Given a couple $\left(X_{1}, X_{2}\right)$ of compatible Banach spaces $\left(X_{i},\|\cdot\|_{i}\right)(i=1,2)$, that is there exists a topological vector space $X$ such that $X_{i} \subset X, i=1,2$, and $X_{1} \cap X_{2} \neq \emptyset$, the corresponding $K$-functional of $x \in X_{1}+X_{2}$ is defined by

$$
K\left(x, t ; X_{1}, X_{2}\right):=\inf \left\{\left\|x_{1}\right\|_{1}+t\left\|x_{2}\right\|_{2}: x=x_{1}+x_{2}, x_{i} \in X_{i}(i=1,2)\right\}
$$

for $t>0$.

If $x$ is a real number, then $\lfloor x\rfloor$ denotes its integer part. As usual, $\log x$ denotes the natural logarithm of a positive real number $x$. The logarithm of $x>0$ to the base 2 is denoted by $\log _{2}(x)$.

Given two positive quantities $X$ and $Y$, if there exists a positive constant $C>0$ such that $X \leq C Y$, we shall write $X \lesssim Y$. If the constant $C$ depends on some parameters $s_{1}, \ldots, s_{n}$, then we shall also write $X \lesssim_{s_{1}, \ldots, s_{n}}, Y$. Moreover, if $X \lesssim Y$ and $Y \lesssim X$, we write $X \sim Y$.

2. Proof of Theorem 1. Let $T$ be a sublinear operator satisfying (1.5) and let $f$ be a fixed analytic trigonometric polynomial on $\mathbb{T}$. We shall prove that

$$
\|T(f)\|_{L^{1}(\mathbb{T})} \leq A+B \int_{\mathbb{T}}|f(\theta)| \log ^{r}(1+|f(\theta)|) d \theta,
$$

where $A, B>0$ depend only on $C_{0}, r$ and not on $f$. Towards this aim, following [16] (see also [15]), for $\lambda>0$, consider the function

$$
a_{\lambda}(\theta):=\max \left\{1,\left(\frac{|f(\theta)|}{\lambda}\right)^{1 / 3}\right\}
$$

and then define

$$
F_{\lambda}(\theta):=\frac{1}{a_{\lambda}(\theta)+i H\left(a_{\lambda}\right)(\theta)}
$$

and

$$
G_{\lambda}(\theta):=1-\left(1-\left[F_{\lambda}(\theta)\right]^{4}\right)^{4}
$$


Here, $H$ denotes the periodic Hilbert transform. It follows that $F_{\lambda}, G_{\lambda} \in$ $H^{\infty}(\mathbb{T})$; see e.g. the proof of $\left[22\right.$, Lemma 7.4.2]. Moreover, since $\left|F_{\lambda}\right| \leq$ $\min \left\{1, \lambda^{1 / 3}|f|^{-1 / 3}\right\}$, one has

$$
\left|G_{\lambda}\right|=\left|1-\left(1-\left[F_{\lambda}\right]^{4}\right)^{4}\right| \leq A_{0}\left|F_{\lambda}\right|^{4} \leq A_{0}\left|F_{\lambda}\right|^{3} \leq A_{0} \min \left\{1, \lambda|f|^{-1}\right\},
$$

where $A_{0}>0$ is an absolute constant. In particular, $\left|G_{\lambda} f\right| \lesssim \lambda$ on $\mathbb{T}$ and so, in order to define an appropriate "bounded analytic replacement" of $\chi_{\{\lambda \leq|f|<2 \lambda\}} f$, one is led to consider functions of the form $\left(G_{2 \lambda}-G_{\lambda}\right) f$. More precisely, arguing as in the proof of [16, Lemma 4.2$]$, consider the functions $\left(\tilde{f}_{n}\right)_{n \in \mathbb{N}_{0}}$ in $H^{\infty}(\mathbb{T})$ given by

$$
\widetilde{f_{0}}:=G_{1} f
$$

and

$$
\widetilde{f}_{n}:=\left(G_{2^{n}}-G_{2^{n-1}}\right) f
$$

for $n \in \mathbb{N}$. Note that there exists an $N \in \mathbb{N}$, depending on $f$, such that $\widetilde{f}_{k} \equiv 0$ for all $k \geq N$. Indeed, since $f$ is an analytic trigonometric polynomial, if we take $N \in \mathbb{N}$ such that $2^{N-1}>\|f\|_{L^{\infty}(\mathbb{T})}$, then for every $k \geq N$, one has $|f(\theta)|<2^{k-1}$ for all $\theta \in \mathbb{T}$. Hence, for every $k \geq N$, one has $a_{2^{k-1}}=a_{2^{k}} \equiv 1$ on $\mathbb{T}$. Therefore, $G_{2^{k-1}}=G_{2^{k}} \equiv 1$ on $\mathbb{T}$ and we thus deduce that $\tilde{f}_{k}=$ $\left(G_{2^{k}}-G_{2^{k-1}}\right) f \equiv 0$ on $\mathbb{T}$ whenever $k \geq N$. So, one has the decomposition

$$
f(\theta)=\sum_{n=0}^{N} \widetilde{f}_{n}(\theta)
$$

for all $\theta \in \mathbb{T}$. Next, as in Yano's paper [27], using the sublinearity of $T$ and then Hölder's inequality, one deduces that

$$
\|T(f)\|_{L^{1}(\mathbb{T})} \leq \sum_{n=0}^{N}\left\|T\left(\widetilde{f}_{n}\right)\right\|_{L^{p_{n}(\mathbb{T})}},
$$

where $p_{n}=1+1 /(n+1)$. Hence, using our assumption (1.5) for each $0 \leq n \leq$ $N$, one gets

$$
\|T(f)\|_{L^{1}(\mathbb{T})} \leq C_{0} \sum_{n=0}^{N}(n+1)^{r}\left\|\widetilde{f}_{n}\right\|_{L^{p_{n}(\mathbb{T})}} .
$$

For $1 \leq n \leq N$, note that since

$$
\left|\tilde{f}_{n}\right| \leq\left|G_{2^{n}}\right||f|+\left|G_{2^{n-1}}\right||f|
$$

it follows from (2.2) that

$$
\left|\widetilde{f}_{n}\right| \leq A_{0}^{\prime} 2^{n}
$$


where $A_{0}^{\prime}>0$ is an absolute constant, independent of $f$ and $n$. Hence, for $1 \leq n \leq N$, we have

$$
\begin{aligned}
\left\|\tilde{f}_{n}\right\|_{L^{p_{n}(\mathbb{T})}} & =A_{0}^{\prime} 2^{n}\left(\int_{\mathbb{T}}\left[\left(A_{0}^{\prime} 2^{n}\right)^{-1}\left|\tilde{f}_{n}(\theta)\right|\right]^{\frac{n+2}{n+1}} d \theta\right)^{\frac{n+1}{n+2}} \\
& \leq A_{0}^{\prime} 2^{n}\left(\int_{\mathbb{T}}\left(A_{0}^{\prime} 2^{n}\right)^{-1}\left|\tilde{f}_{n}(\theta)\right| d \theta\right)^{\frac{n+1}{n+2}}
\end{aligned}
$$

and we thus deduce that

$$
\left\|\widetilde{f}_{n}\right\|_{L^{p_{n}(\mathbb{T})}} \lesssim\left(\int_{\mathbb{T}}\left|\tilde{f}_{n}(\theta)\right| d \theta\right)^{\frac{n+1}{n+2}}
$$

where the implied constant is independent of $n$. Using now the elementary inequality

$$
t^{(n+1) /(n+2)} \leq e^{r+2} t+(n+1)^{-(r+2)},
$$

which is valid for all $t>0$ and $n \geq 0$, we obtain

$$
\left\|\widetilde{f}_{n}\right\|_{L^{p_{n}(\mathbb{T})}} \lesssim r \int_{\mathbb{T}}\left|\tilde{f}_{n}(\theta)\right| d \theta+\frac{1}{(n+1)^{r+2}},
$$

where the implied constant depends only on $r$ and not on $f, n$. Moreover, note that by using (2.2) for $\lambda=1$, one has

$$
\left\|\widetilde{f}_{0}\right\|_{L^{1}(\mathbb{T})} \lesssim 1
$$

Hence, it follows from (2.3), (2.5), and (2.6) that

$$
\|T(f)\|_{L^{1}(\mathbb{T})} \lesssim C_{0}, r 1+\sum_{n=1}^{N}(n+1)^{r}\left\|\widetilde{f}_{n}\right\|_{L^{1}(\mathbb{T})}
$$

Therefore, to prove (2.1), it suffices to show that

$$
\sum_{n=1}^{N}(n+1)^{r}\left\|\widetilde{f}_{n}\right\|_{L^{1}(\mathbb{T})} \lesssim_{r} 1+\int_{\mathbb{T}}|f(\theta)| \log ^{r}(1+|f(\theta)|) d \theta .
$$

To this end, the idea is to write

$$
\left\|\widetilde{f}_{n}\right\|_{L^{1}(\mathbb{T})}=\int_{\left\{|f|<2^{n-1}\right\}}\left|\tilde{f}_{n}(\theta)\right| d \theta+\int_{\left\{|f| \geq 2^{n-1}\right\}}\left|\tilde{f}_{n}(\theta)\right| d \theta=I_{n}^{(1)}+I_{n}^{(2)}
$$

and then treat each of the terms $I_{n}^{(1)}, I_{n}^{(2)}$ separately showing that

$$
\sum_{n=1}^{N}(n+1)^{r} I_{n}^{(i)} \lesssim r 1+\int_{\mathbb{T}}|f(\theta)| \log ^{r}(1+|f(\theta)|) d \theta \quad(i=1,2) .
$$

Note that (2.8) (for $i=1,2$ ) automatically establishes (2.7). We have thus reduced matters to showing $(2.8)$ for $i=1,2$. 
The case $i=2$ in (2.8) is the easiest one; using (2.4) for each $1 \leq n \leq N$, we get

$$
\begin{aligned}
\sum_{n=1}^{N}(n+1)^{r} I_{n}^{(2)} & =\sum_{n=1}^{N}(n+1)^{r} \int_{\left\{|f| \geq 2^{n-1}\right\}}\left|\tilde{f}_{n}(\theta)\right| d \theta \\
& \lesssim \sum_{n=1}^{N}(n+1)^{r} \int_{\left\{|f| \geq 2^{n-1}\right\}} 2^{n} d \theta .
\end{aligned}
$$

Hence, by using Fubini's theorem, we obtain

$$
\begin{aligned}
\sum_{n=1}^{N}(n+1)^{r} I_{n}^{(2)} & \lesssim \int_{\mathbb{T}}\left(\sum_{n=1}^{2+\left\lfloor\log _{2}(|f(\theta)|+1)\right\rfloor} 2^{n}(n+1)^{r}\right) d \theta \\
& \lesssim r 1+\int_{\mathbb{T}}|f(\theta)| \log ^{r}(1+|f(\theta)|) d \theta,
\end{aligned}
$$

as desired.

It remains to prove $(2.8)$ for $i=1$. Towards this aim, note that since

$$
G_{2^{n}}-G_{2^{n-1}}=\left(1-\left[F_{2^{n}-1}\right]^{4}\right)^{4}-\left(1-\left[F_{2^{n}}\right]^{4}\right)^{4}
$$

and $\left|F_{2^{n}-1}\right| \leq 1,\left|F_{2^{n}}\right| \leq 1$ on $\mathbb{T}$, it follows that

$$
\left|G_{2^{n}}-G_{2^{n-1}}\right| \leq\left|1-\left[F_{2^{n}}\right]^{4}\right|^{4}+\left|1-\left[F_{2^{n}-1}\right]^{4}\right|^{4} \lesssim\left|1-F_{2^{n}}\right|^{2}+\left|1-F_{2^{n-1}}\right|^{2}
$$

on $\mathbb{T}$, where the implied constant is independent of $n$. Hence, we have

$$
\begin{aligned}
I_{n}^{(1)} & =\int_{\left\{|f|<2^{n-1}\right\}}\left|\tilde{f}_{n}(\theta)\right| d \theta \\
& \lesssim \int_{\left\{|f|<2^{n-1}\right\}}\left|1-F_{2^{n}}(\theta)\right|^{2}|f(\theta)| d \theta+\int_{\left\{|f|<2^{n-1}\right\}}\left|1-F_{2^{n-1}}(\theta)\right|^{2}|f(\theta)| d \theta \\
& \leq I_{n}^{(1, \alpha)}+I_{n}^{(1, \beta)}
\end{aligned}
$$

where

$$
I_{n}^{(1, \alpha)}:=2^{n-1} \int_{\left\{|f|<2^{n-1}\right\}}\left|1-F_{2^{n}}(\theta)\right|^{2} d \theta
$$

and

$$
I_{n}^{(1, \beta)}:=2^{n-1} \int_{\left\{|f|<2^{n-1}\right\}}\left|1-F_{2^{n-1}}(\theta)\right|^{2} d \theta .
$$

We shall prove that

$$
I_{n}^{(1, \alpha)} \lesssim 2^{n / 3} \int|f(\theta)|^{2 / 3} d \theta
$$


and

$$
I_{n}^{(1, \beta)} \lesssim 2^{n / 3} \int_{\left\{|f| \geq 2^{n-1}\right\}}|f(\theta)|^{2 / 3} d \theta .
$$

To prove (2.9), we shall again make use of arguments from [16] (see also [15]); since the periodic Hilbert transform $H$ of any constant function on $\mathbb{T}$ is identically 0 , one may write

$$
I_{n}^{(1, \alpha)}=2^{n-1} \int_{\left\{|f|<2^{n-1}\right\}}\left|\frac{\left(1-a_{2^{n}}(\theta)\right)+i H\left(1-a_{2^{n}}\right)(\theta)}{a_{2^{n}}(\theta)+i H\left(a_{2^{n}}\right)(\theta)}\right|^{2} d \theta
$$

and since $a_{2^{n}} \equiv 1$ on $\left\{|f|<2^{n-1}\right\}$, it follows that

$$
I_{n}^{(1, \alpha)} \leq 2^{n-1} \int_{\left\{|f|<2^{n-1}\right\}}\left|H\left(1-a_{2^{n}}\right)(\theta)\right|^{2} d \theta \leq 2^{n-1}\left\|H\left(1-a_{2^{n}}\right)\right\|_{L^{2}(\mathbb{T})}^{2} .
$$

Hence, by using the $L^{2}$-boundedness of $H$, one gets

$$
\begin{aligned}
I_{n}^{(1, \alpha)} \leq 2^{n-1}\left\|1-a_{2^{n}}\right\|_{L^{2}(\mathbb{T})}^{2} & =2^{n-1} \int_{\left\{|f| \geq 2^{n}\right\}}\left|\left(\frac{|f(\theta)|}{2^{n}}\right)^{1 / 3}-1\right|^{2} d \theta \\
& \leq 2^{n / 3} \int_{\left\{|f| \geq 2^{n}\right\}}|f(\theta)|^{2 / 3} d \theta
\end{aligned}
$$

and this completes the proof of (2.9). The proof of (2.10) is completely analogous. It thus follows from (2.9) and (2.10) that

$$
I_{n}^{(1)} \lesssim 2^{n / 3} \int_{\left\{|f| \geq 2^{n-1}\right\}}|f(\theta)|^{2 / 3} d \theta
$$

and hence, by using Fubini's theorem, we have

$$
\begin{aligned}
\sum_{n=1}^{N}(n+1)^{r} I_{n}^{(1)} & \lesssim \int_{\mathbb{T}}|f(\theta)|^{2 / 3}\left(\sum_{n=1}^{2+\left\lfloor\log _{2}(|f(\theta)|+1)\right\rfloor} 2^{n / 3}(n+1)^{r}\right) d \theta \\
& \lesssim_{r} 1+\int_{\mathbb{T}}|f(\theta)| \log ^{r}(1+|f(\theta)|) d \theta .
\end{aligned}
$$

Therefore, the proof of (2.8) for $i=1$ is complete. So, (2.8) holds for $i=1,2$ and hence, (2.7) is also true. We have thus shown that (2.1) holds for every analytic trigonometric polynomial on the torus.

To complete the proof of Theorem 1, note that (1.6) follows directly from (2.1) using a simple scaling argument. Indeed, if $f$ is an analytic trigonometric polynomial on $\mathbb{T}$ with $\|f\|_{L \log ^{r} L(\mathbb{T})}=1$, then $\int_{\mathbb{T}}|f(\theta)| \log ^{r}(1+|f(\theta)|) d \theta \lesssim_{r} 1$. Hence, one deduces from (2.1) that if $\|f\|_{L \log ^{r} L(\mathbb{T})}=1$, then $\|T(f)\|_{L^{1}(\mathbb{T})} \leq D$, where $D>0$ is an absolute constant depending only on $C_{0}, r$. In the general case, if $f$ is a non-zero analytic trigonometric polynomial on $\mathbb{T}$, then, using the previous implication and the scaling invariance of $T$, (1.6) follows. 


\section{Some further remarks.}

3.1. Sharpness of Theorem 1. As mentioned in the introduction, Zygmund's inequality (1.3) on the classical Littlewood-Paley square function can now be obtained as a corollary of Pichorides's result (1.4) via Theorem 1. Moreover, the exponent $r=1$ in the Orlicz space $L \log ^{r} L(\mathbb{T})$ in (1.3) cannot be improved; see [2]. Therefore, the example of the Littlewood-Paley square function $S$ shows that Theorem 1 is in general sharp in the following sense; there exists a sublinear operator $T$ and an $r=r(T)>0$ such that $\|T\|_{\left(H^{p}(\mathbb{T}),\|\cdot\|_{L^{p}(\mathbb{T})}\right) \rightarrow\left(L^{p}(\mathbb{T}),\|\cdot\|_{L^{p}(\mathbb{T})}\right)}$ $\sim(p-1)^{-r}$ as $p \rightarrow 1^{+}$and $\|T(f)\|_{L^{1}(\mathbb{T})} \lesssim\|f\|_{L \log ^{s} L(\mathbb{T})}$ holds for every analytic trigonometric polynomial $f$ when $s=r$ but it does not hold for any exponent $s<r$. In particular, for the Littlewood-Paley square function $S$ one has $r=r(S)=1$.

Note that if one removes the analyticity assumptions, then the behaviour of $S$ "near" $L^{1}(\mathbb{T})$ is different than the one mentioned above. In particular, Bourgain showed in [8] that the $L^{p}(\mathbb{T}) \rightarrow L^{p}(\mathbb{T})$ operator norm of $S$ behaves like $(p-1)^{-3 / 2}$ as $p \rightarrow 1^{+}$. Moreover, $f \in L \log ^{3 / 2} L(\mathbb{T})$ implies that $S(f)$ is in $L^{1}(\mathbb{T})$ and the exponent $r=3 / 2$ in $L \log ^{3 / 2} L(\mathbb{T})$ is the best possible; see [1].

Another example illustrating Theorem 1 and its sharpness (in the sense discussed above) is given by some classical results of Y. Meyer [20] and A. Bonami [5]. More specifically, consider the following subset of positive integers

$$
\Lambda=\left\{3^{k}-3^{m}: 0 \leq m \leq k-1 \text { and } k, m \in \mathbb{Z}\right\} .
$$

Then [5, Corollaire 4] asserts that there exists an absolute constant $C>0$ such that for every $q>2$, one has

$$
\|h\|_{L^{q}(\mathbb{T})} \leq C q\|h\|_{L^{2}(\mathbb{T})}
$$

for all trigonometric polynomials $h$ on $\mathbb{T}$ with $\operatorname{supp}(\widehat{h}) \subset \Lambda$. Hence, if we consider the multiplier operator $T_{\Lambda}$ on $\mathbb{T}$ with symbol $\chi_{\Lambda}$, namely for every trigonometric polynomial $f$, one has

$$
T_{\Lambda}(f)(\theta)=\sum_{n \in \Lambda} \widehat{f}(n) e^{i 2 \pi n \theta}
$$

for all $\theta \in \mathbb{T}$, then it follows from (3.1) and duality that $T_{\Lambda}$ satisfies

$$
\sup _{\substack{g \in L^{p}(\mathbb{T}): \\\|g\|_{L^{p}(\mathbb{T})}=1}}\left\|T_{\Lambda}(g)\right\|_{L^{p}(\mathbb{T})} \lesssim(p-1)^{-1} \quad\left(\text { as } p \rightarrow 1^{+}\right)
$$

and

$$
\left\|T_{\Lambda}(f)\right\|_{L^{1}(\mathbb{T})} \lesssim\|f\|_{L \log L(\mathbb{T})}
$$

However, if we restrict ourselves to analytic Hardy spaces on the torus, then it follows from parts (a) and (c) of [20, pp. 549-550, Théorème 1] that one has the improved bounds

$$
\sup _{\substack{g \in H^{p}(\mathbb{T}): \\\|g\|_{L^{p}(\mathbb{T})}=1}}\left\|T_{\Lambda}(g)\right\|_{L^{p}(\mathbb{T})} \lesssim(p-1)^{-1 / 2} \quad\left(\text { as } p \rightarrow 1^{+}\right)
$$


and

$$
\left\|T_{\Lambda}(f)\right\|_{L^{1}(\mathbb{T})} \lesssim\|f\|_{L \log ^{1 / 2} L(\mathbb{T})}\left(f \in H^{1}(\mathbb{T})\right),
$$

respectively. In particular, the linear operator $T_{\Lambda}$ satisfies (1.5) and (1.6) in Theorem 1 for $r=1 / 2$.

Furthermore, the exponents $r=1 / 2$ in $(p-1)^{-1 / 2}$ in $(3.4)$ and $r=1 / 2$ in $L \log ^{1 / 2} L(\mathbb{T})$ in $(3.5)$ cannot be improved. Indeed, to see that (3.5) is sharp, note that, by using (3.1) and [25, (1.4.1)], one has

$$
\left\|T_{\Lambda}(f)\right\|_{L^{1}(\mathbb{T})} \leq\left\|T_{\Lambda}(f)\right\|_{L^{2}(\mathbb{T})} \lesssim\left\|T_{\Lambda}(f)\right\|_{L^{1}(\mathbb{T})}
$$

for every trigonometric polynomial $f$ on $\mathbb{T}$. Assume now that for some $r>0$, one has

$$
\left\|T_{\Lambda}(f)\right\|_{L^{1}(\mathbb{T})} \lesssim\|f\|_{L \log ^{r} L(\mathbb{T})}\left(f \in H^{1}(\mathbb{T})\right)
$$

To show that $r \geq 1 / 2$, for a large $N \in \mathbb{N}$ that will eventually be sent to infinity, consider the function $\beta_{N} \in H^{\infty}(\mathbb{T})$ given by

$$
\beta_{N}(\theta)=e^{i 2 \pi\left(2 \cdot 3^{N}+1\right) \theta} V_{3^{N}}(\theta),
$$

where $V_{n}=2 K_{2 n+1}-K_{n}$ is the de la Vallée Poussin kernel of order $n \in \mathbb{N}$ and $K_{n}$ denotes the $n$-th Féjer kernel, i.e. $K_{n}(\theta)=\sum_{|j| \leq n}[1-|j| /(n+1)] e^{i 2 \pi j \theta}$, $\theta \in \mathbb{T}$. Since $\left\|\beta_{N}\right\|_{L \log ^{r} L(\mathbb{T})} \lesssim N^{r}$ and $\widehat{\beta_{N}}(j)=1$ for all $j \in \mathbb{N}$ with $3^{N} \leq j \leq$ $3^{N+1}+2$, it follows from (3.7) and (3.6) that

$$
\begin{aligned}
N^{r} \gtrsim\left\|\beta_{N}\right\|_{L \log ^{r} L(\mathbb{T})} \gtrsim\left\|T_{\Lambda}\left(\beta_{N}\right)\right\|_{L^{1}(\mathbb{T})} & \gtrsim\left\|T_{\Lambda}\left(\beta_{N}\right)\right\|_{L^{2}(\mathbb{T})} \\
& =\left(\sum_{k=1}^{N+2} \sum_{m=0}^{k-1}\left|\widehat{\beta_{N}}\left(3^{k}-3^{m}\right)\right|^{2}\right)^{1 / 2} \\
& \geq\left(\sum_{m=0}^{N}\left|\widehat{\beta_{N}}\left(3^{N+1}-3^{m}\right)\right|^{2}\right)^{1 / 2} \\
& \sim N^{1 / 2}
\end{aligned}
$$

and so, by taking $N \rightarrow \infty$, one deduces that $r \geq 1 / 2$, i.e. (3.5) is sharp. Similarly, one shows that the exponent $r=1 / 2$ in $(p-1)^{-1 / 2}$ in (3.4) as well as the exponents $r=1$ in $(p-1)^{-1}$ in (3.2) and $r=1$ in $L \log L(\mathbb{T})$ in (3.3) are best possible.

3.2. Extension of Theorem 1 to Hardy-Orlicz spaces. If $T$ satisfies the assumptions of Theorem 1 and, moreover, $|T(f)-T(g)| \leq|T(f-g)|$ holds, then one can easily show that (1.6) can be extended to all functions $f$ such that $\|f\|_{L \log ^{r} L(\mathbb{T})}<\infty$ and $\operatorname{supp}(\widehat{f}) \subset \mathbb{N}_{0}$. To this end, one adapts e.g. the density argument on [30, Chapter XII, p. 120]. More precisely, fix a function $f$ with $\|f\|_{L \log ^{r} L(\mathbb{T})}<\infty$ and $\operatorname{supp}(\widehat{f}) \subset \mathbb{N}_{0}$. By [19, Proposition 3.4], there exists a sequence of analytic trigonometric polynomials $\left(\phi_{n}\right)_{n \in \mathbb{N}}$ that converges to $f$ in $\left(L \log ^{r} L(\mathbb{T}),\|\cdot\|_{L \log ^{r} L(\mathbb{T})}\right)$. It thus follows from (1.6) that the sequence $\left(T\left(\phi_{n}\right)\right)_{n \in \mathbb{N}}$ is Cauchy in $L^{1}(\mathbb{T})$ and so, it converges to some $g \in L^{1}(\mathbb{T})$ and 
$g$ is independent of the choice of $\left(\phi_{n}\right)_{n \in \mathbb{N}}$. Hence, if we set $T(f):=g$, we deduce that (1.6) holds for $f$. Therefore, following the terminology of [19], $T$ is uniquely extended as a bounded operator from the Orlicz-Hardy space $H^{\Phi_{r}}(\mathbb{T})$ to $L^{1}(\mathbb{T})$, where $\Phi_{r}(x)=x\left([1+\log (x+1)]^{r}-1\right), x \geq 0$.

3.3. Higher-dimensional variants. In [2], both the results of Zygmund and Pichorides were extended to higher dimensions. Hence, in analogy to the onedimensional case, one is naturally led to ask whether versions of Theorem 1 involving Hardy spaces of several variables hold as well.

At this point, it is worth mentioning that the methods used here are closely connected with the $K$-closedness of $\left(H^{1}(\mathbb{T}), H^{\infty}(\mathbb{T})\right)$ with respect to $\left(L^{1}(\mathbb{T}), L^{\infty}(\mathbb{T})\right)$, a result that follows from the work of Peter Jones [14]. To be more specific, the proof presented in Section 2 is related to the fact that for every $f \in H^{1}(\mathbb{T})+H^{\infty}(\mathbb{T})$ and $t \in[0,1]$, one has

$$
K\left(f, t ; H^{1}(\mathbb{T}), H^{\infty}(\mathbb{T})\right) \sim K\left(f, t ; L^{1}(\mathbb{T}), L^{\infty}(\mathbb{T})\right)=\int_{0}^{t} f^{*}(s) d s
$$

in the sense that our argument is based on Kislyakov's "analytic decomposition of unity", a technique that was used by Xu in [28] to obtain another proof of (3.8). For other proofs of the $K$-closedness of $\left(H^{1}(\mathbb{T}), H^{\infty}(\mathbb{T})\right)$ with respect to $\left(L^{1}(\mathbb{T}), L^{\infty}(\mathbb{T})\right)$, see P. Müller [21], G. Pisier [24] as well as Bourgain [9]. Furthermore, as mentioned in the introduction, Theorem 1 also follows from the work of Carro and Martín [11] combined with (3.8). More precisely, a proof of Theorem 1 can be obtained by using [11, Theorem 3.1] combined with (1.7), (3.8), and "Hardy's lemma"; see [4, p. 56, Proposition 3.6].

Similarly, if $n=2$, then it was shown by Kislyakov and $\mathrm{Xu}$ that the couple $\left(H^{1}\left(\mathbb{T}^{2}\right), H^{\infty}\left(\mathbb{T}^{2}\right)\right)$ is $K$-closed with respect to $\left(L^{1}\left(\mathbb{T}^{2}\right), L^{\infty}\left(\mathbb{T}^{2}\right)\right)$; see $[17$, Theorem 3] and hence, by arguing as above, one obtains a two-dimensional version of Theorem 1 . However, to the best of our knowledge, the $K$-closedness of $\left(H^{1}\left(\mathbb{T}^{n}\right), H^{\infty}\left(\mathbb{T}^{n}\right)\right)$ with respect to $\left(L^{1}\left(\mathbb{T}^{n}\right), L^{\infty}\left(\mathbb{T}^{n}\right)\right)$ is still an open problem for $n \geq 3$ and so, in view of the aforementioned, already existing results and the remarks made above, it seems that a version of Theorem 1 for $n \geq 3$ is not yet available.

Acknowledgements. The author would like to thank Salvador Rodríguez-López and Alan Sola for some interesting discussions and for carefully reading earlier versions of this manuscript.

Open Access. This article is distributed under the terms of the Creative Commons Attribution 4.0 International License (http://creativecommons.org/licenses/ by/4.0/), which permits unrestricted use, distribution, and reproduction in any medium, provided you give appropriate credit to the original author(s) and the source, provide a link to the Creative Commons license, and indicate if changes were made.

Publisher's Note Springer Nature remains neutral with regard to jurisdictional claims in published maps and institutional affiliations. 


\section{References}

[1] Bakas, O.: Endpoint mapping properties of the Littlewood-Paley square function. Colloq. Math. 157(1), 1-15 (2019)

[2] Bakas, O., Rodríguez-López, S., Sola, A.: Multi-parameter extensions of a theorem of Pichorides. Proc. Am. Math. Soc. 147(3), 1081-1095 (2019)

[3] Bennett, C., Rudnick, K.: On Lorentz-Zygmund spaces. Diss. Math. 175, 67 pp (1980)

[4] Bennett, C., Sharpley, R.: Interpolation of Operators. Pure and Applied Mathematics, vol. 129. Academic Press, Boston (1988)

[5] Bonami, A.: Étude des coefficients de Fourier des fonctions de $L^{p}(G)$. Ann. Inst. Fourier (Grenoble) 20(2), 335-402 (1970)

[6] Bourgain, J.: New Banach space properties of the disc algebra and $H^{\infty}$. Acta Math. 152(1-2), 1-48 (1984)

[7] Bourgain, J.: Bilinear forms on $H^{\infty}$ and bounded bianalytic functions. Trans. Am. Math. Soc. 286(1), 313-337 (1984)

[8] Bourgain, J.: On the behavior of the constant in the Littlewood-Paley inequality. In: Geometric Aspects of Functional Analysis (1987-1988). Lecture Notes in Mathematics, 1376, pp. 202-208. Springer, Berlin (1989)

[9] Bourgain, J.: Some consequences of Pisier's approach to interpolation. Isr. J. Math. 77(1-2), 165-185 (1992)

[10] Brudnyi, Y., Kruglyak, N.Y.: Interpolation Functors and Interpolation Spaces. North-Holland Mathematical Library, 47, vol. I, xvi+718 pp. North-Holland Publishing Co., Amsterdam (1991)

[11] Carro, M.J., Joaquim, Martín: Extrapolation theory for the real interpolation method. Collect. Math. 53(2), 165-186 (2002)

[12] Duren, P.L.: Theory of $H^{p}$ Spaces. Pure and Applied Mathematics, vol. 38. Academic Press, New York, xii+258 pp (1970)

[13] Jawerth, B., Milman, M.: Extrapolation theory with applications. Mem. Amer. Math. Soc. 89 (440), iv+82 pp (1991)

[14] Jones, P.W.: $L^{\infty}$ estimates for the $\bar{\partial}$ problem in a half-plane. Acta Math. 150(12), 137-152 (1983)

[15] Kislyakov, S.V.: Absolutely summing operators on the disc algebra. Algebra i Analiz 3(4), 1-77 (1991); translation in St. Petersburg Math. J. 3(4), 705-774 (1992)

[16] Kislyakov, S.V., Xu, Q.: Interpolation of weighted and vector-valued Hardy spaces. Trans. Am. Math. Soc. 343(1), 1-34 (1994)

[17] Kislyakov, S.V., Xu, Q.: Real interpolation and singular integrals. Algebra i Analiz 8(4), 75-109 (1996). translation in St. Petersburg Math. J. 8(4), 593-615 (1997)

[18] Krasnosel'skii, M.A., Rutitskii, Y.B.: Convex functions and Orlicz spaces. P. Noordhoff, Groningen (1961) 
[19] Lefèvre, P., Li, D., Queffélec, H., Rodríguez-Piazza, L.: Composition operators on Hardy-Orlicz spaces. Mem. Amer. Math. Soc. 207(974), vi+74 pp (2010)

[20] Meyer, Y.: Endomorphismes des idéaux fermés de $L^{1}(G)$, classes de Hardy et séries de Fourier lacunaires. Ann. Sci. École Norm. Sup. (4), 1, 499-580 (1968)

[21] Müller, P.F.X.: Holomorphic martingales and interpolation between Hardy spaces. J. Anal. Math. 61(1), 327-337 (1993)

[22] Pavlović, M.: Introduction to Function Spaces on the Disk. Posebna Izdanja [Special editions] 20, Matematicki Institut SANU, Belgrade (2004)

[23] Pichorides, S.K.: A remark on the constants of the Littlewood-Paley inequality. Proc. Am. Math. Soc. 114(3), 787-789 (1992)

[24] Pisier, G.: Interpolation between $H^{p}$ spaces and noncommutative generalizations. I. Pac. J. Math. 155(2), 341-368 (1992)

[25] Rudin, W.: Trigonometric series with gaps. J. Math. Mech. 9, 203-227 (1960)

[26] Tao, T., Wright, J.: Endpoint multiplier theorems of Marcinkiewicz type. Rev. Mat. Iberoam. 17(3), 521-558 (2001)

[27] Yano, S.: Notes on fourier analysis (XXIX): an extrapolation theorem. J. Math. Soc. Japan 3(2), 296-305 (1951)

[28] Xu, Q.: Notes on interpolation of Hardy spaces. Ann. Inst. Fourier 42(4), 875889 (1992)

[29] Zygmund, A.: On the convergence and summability of power series on the circle of convergence (I). Fund. Math. 30, 170-196 (1938)

[30] Zygmund, A.: Trigonometric Series, vol. I, II. Cambridge University Press, Cambridge (2002)

ODYSSEAS BAKAS

Department of Mathematics

Stockholm University

10691 Stockholm

Sweden

e-mail: bakas@math.su.se

Received: 30 September 2018 